It is an ideal example of a mismatch in

understanding with no-one saying

anything but both sides making incorrect

assumptions.

\title{
Mismatch in understanding
}

In the News column in this issue we are running a story about a survey carried out by a commercial company into the views of both dentists and patients on the availability of cosmetic dentistry.

The survey was sent to 125 dental practices in Gloucestershire, focusing on practices with over 1,500 active patients, with $60 \%$ of practices treating patients under private contract and the remaining 40\% seeing patients on both a private and NHS basis. The response rate was a respectable $72 \%$. The company also questioned several hundred patients about their views on cosmetic dentistry.

It appears that over $70 \%$ of the dentists surveyed said they would prefer to focus more on cosmetic dentistry, yet could not because they were too busy with general dentistry. At the same time $63 \%$ of the patients surveyed said they would be prepared to consider cosmetic dentistry, and more revealingly they would be prepared to pay on average around $£ 700$ for a course of treatment. Some said they would be prepared to spend more if the payments could be spread.

Given the facts above, one is tempted to ask the question "What's the problem then?" The patients, it appears, want more cosmetic dentistry and are prepared to pay for it. The dentists want to carry out more cosmetic dentistry. Yet is is not happening.

I can think of two possible reasons for this. Either the patients do not like to ask the 'busy dentist' if they can have cosmetic dentistry, or perhaps more disturbingly think the dentist is incapable of providing it - because if they were capable then surely they would suggest it. In fact the survey of patients mentions this very point, which is a worrying one for the profession. Meanwhile, the dentists, either because they are too busy or because they do not want to feel they are 'pressurising' the patients wait for the patient to ask for cosmetic dentistry, working on the assumption that if they really wanted it then they would mention it themselves. It is an ideal example of a mismatch in understanding with no-one saying anything but both sides making incorrect assumptions.
It is interesting to speculate how often this happens in other professions or situations. I can think of parallels in the world of financial advice (where clients are not aware of what can be done for them and advisors are afraid of being accused of aggressive selling) but it is not really the same. In the example in dentistry the patients know what they want but do not feel it is appropriate to ask. For some reason they feel inhibited in the dental environment.

Of course we know that people feel ill at ease at the dentist's, and this survey has pinpointed yet again how important it is for everyone in the practice to work hard at overcoming the barriers to good communication. This is a well-worn theme, but no less important for that, and things are improving.

But the other side of the communication mismatch is even more perplexing. Why, based on this survey, are so many dentists failing to offer patients the treatment that the patients want and the dentists want to do? Why do they allow the fact they are so busy prevent them from offering cosmetic dentistry? Given the fact the these practices offer more dental care privately we must assume it is nothing to do with the current NHS regulations.

I could speculate on the answers, and I suspect the real answers vary according to the practice, the patients and the dentists themselves, but perhaps there is a more universal reason linked to the historical patterns of behaviour in general practice over the last 50 years. Could it be that the main system of remuneration in dental practice, a fee-per-item system, has created a culture of 'busyness' where not working at full tilt creates a feeling of guilt and a concern the dentist is somehow not being successful?

The survey has highlighted something I suspect we already knew. It seems many of our patients want more cosmetic dentistry and are prepared to pay for it. We just have to remember to suggest it to them.

Mike Grace m.grace@bda-dentistry.org.uk 